Expansi on shock waves i $\mathrm{n}$ the i mpl osi on process from a ti me-rever si bl e mol ecul ar-dynamics si mul at $i$ on of a dual expl osi on process

\begin{tabular}{|l|l|}
\hline 著者 & Konat su Nobuyoshi , Abe Takashi \\
\hline $\begin{array}{l}\text { j our nal or } \\
\text { publ i cat i on t i t l e }\end{array}$ & Physi cs of Fl ui ds \\
\hline vol une & 19 \\
\hline number & 5 \\
\hline page r ange & 056103-1-056103- 6 \\
\hline year & 2007- 05- 01 \\
\hline URL & ht t p: //hdl . handl e. net /2297/6602 \\
\hline
\end{tabular}




\title{
Expansion shock waves in the implosion process from a time-reversible molecular-dynamics simulation of a dual explosion process
}

\author{
Nobuyoshi Komatsu ${ }^{a)}$ and Takashi Abe ${ }^{\text {b) }}$ \\ The Institute of Space and Astronautical Science/JAXA, Yoshinodai 3-1-1, Sagamihara, Kanagawa, Japan
}

(Received 10 July 2006; accepted 26 February 2007; published online 14 May 2007)

\begin{abstract}
Why does not an expansion shock wave exist in a gaseous medium in nature? The reason has been widely believed to be the irreversibility in nature, while an obvious demonstration for this belief has not been accomplished yet. In order to resolve the question from a microscopic viewpoint, an implosion process dual to an explosion process was investigated by means of the molecular-dynamics method (MD). To this aim, we employed a "bit-reversible algorithm (Bit MD)" that was completely time-reversible in a microscopic viewpoint and was free from any round-off error. Here we show that, through a dual implosion simulation (i.e., a time-reversible simulation of the explosion), a kind of expansion shock wave is successfully formed in the Bit MD simulation. Furthermore, we show that when the controlled noise is intentionally added to the Bit MD, the expansion shock wave disappears dramatically and turns into an isentropic expansion wave, even if the noise is extremely small. Since the controlled noise gives rise to the irreversibility in the Bit MD simulation, it can be concluded that the irreversibility in the system prohibits the expansion shock wave from appearing in the system. (C) 2007 American Institute of Physics.
\end{abstract}

[DOI: $10.1063 / 1.2717725$ ]

\section{INTRODUCTION}

Shock waves are a frequently occurring phenomenon in a variety of fields, from a supersonic aircraft to a supernova explosion. Such a shock wave is a so-called "compression shock wave," which compresses a propagation medium after the shock wave passage. On the contrary, it seems that a so-called "expansion shock wave" (or a "rarefied shock wave"), through which a propagation medium expands and becomes less dense while keeping its discontinuity like an ordinary compression shock wave, does not exist in nature, especially for a "gaseous" medium.

First of all, from a macroscopic viewpoint, i.e., from a fluid dynamical point of view, we will review an expansion shock wave. (For instance, details of the expansion shock wave have been discussed in the textbook by Zel'dovich and Raizer. ${ }^{1}$ ) As predicted by Zel'dovich, ${ }^{2}$ analyzed by Thompson, ${ }^{3,4}$ and clarified experimentally by Borisov, ${ }^{5}$ an expansion shock wave can be generated in the vicinity of the "liquid-vapor" critical point, or in some porous solids undergoing a phase transition at high pressures, etc. ${ }^{6-10}$ For example, near the liquid-vapor critical point, the value $\left(\partial^{2} p / \partial v^{2}\right)_{s}$ becomes negative for a substance with a reasonably high specific heat $c_{v}$ (where $p, v$, and $s$ represent the pressure, specific volume, and entropy, respectively) and, therefore, expansion shock waves can be generated under such specific conditions. " In a "gaseous" medium corresponding to normal fluids, however, the value $\left(\partial^{2} p / \partial v^{2}\right)_{s}$ is positive. Accordingly, it is widely believed that the expansion shock wave does not exist in a "gaseous" medium, be-

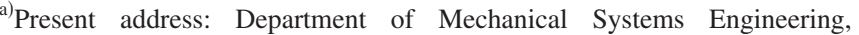
Kanazawa University, Kakuma-machi, Kanazawa 920-1192, Japan. Electronic mail: komatsu@t.kanazawa-u.ac.jp

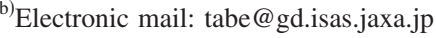

cause of both the second law of thermodynamics (i.e., the law of entropy increase) and its being unstable. ${ }^{1}$ This is because, according to the second law of thermodynamics, which is applicable to macroscopic phenomena, the entropy of a medium cannot be decreased by internal processes alone, without the transfer of heat to an external medium. ${ }^{1}$

However, from a "microscopic" viewpoint, the origin of the second law of thermodynamics or the origin of the irreversibility in nature has not been resolved, ${ }^{11-13}$ since those irreversible macroscopic phenomena should be derived from time-reversible microscopic laws. This is known as the problem of irreversibility: i.e., why does an irreversible behavior of macroscopic systems arise from a time-reversible microscopic law? As a simple example, let us consider a system consisting of a box divided into two regions by a partition. ${ }^{14}$ Suppose that, initially, the left half region is filled with gas composed of molecules and the right half one is empty. Based on our experience, as soon as the partition is removed, the gas expands into the whole container and the molecules never go back to the left half region of the box, although such a motion would be possible because of the timereversible microscopic law. Therefore, to our knowledge, there is no clear evidence why an expansion shock wave does not exist in a gaseous medium, at least from a "microscopic" viewpoint.

The expansion shock wave can be possible at least in a "thought experiment" if we assume that the gas dynamic behavior can be composed of motion of molecules in the gas and the molecular motions are "time-reversible." It seems that this assumption is generally accepted. ${ }^{11-13}$ To illustrate the possibility of the expansion shock wave, we will focus on the duality of explosion and implosion processes. That is, we consider an implosion process that can be generated by the 
time-reversal operation applied, at a certain time, to the ordinary explosion process. In this sense, this imploding process is dual to the explosion process. In the time-reversal operation, velocities of all particles in the gas are reversed instantaneously. In the explosion process, we will observe a kind of shock wave (or a discontinuous wave) propagating outwards. On the other hand, in the dual implosion process, we will observe the discontinuous wave propagating reversely (i.e., inwards in spatial direction) if all the molecules behave in a completely time-reversible manner. In such a discontinuous wave or a kind of shock wave, the propagation medium must expand after the shock passage. Therefore, such a discontinuous wave must be a kind of expansion shock wave. Then, a question arises: Does a law of nature permit such a process? Or if not, how can such a process be avoided in nature? In the present paper, we will answer the question investigating this problem from a microscopic point of view. (Note that, although an imploding shock wave has been investigated by many researchers, ${ }^{15-19}$ the shock wave is not an expansion shock wave but a compression one. Strictly speaking, the shock wave is one of the phenomena occurring in macroscopic fluid dynamics. However, in the present study, the discontinuous wave appearing in microscopic molecular dynamics is also called the shock wave.)

In order to answer the question and to study the expansion shock wave from a microscopic viewpoint, a moleculardynamics method (MD) is quite suitable. Nevertheless, the expansion shock wave has not been investigated so far by means of the MD methods, although the compression shock wave has been studied extensively. ${ }^{20-22}$ We can guess that the reason is both the inherent "irreversibility" of the standard MD methods and the instability of the expansion shock wave. This is because the standard MD methods cannot exactly investigate such unstable systems, due to round-off errors. In other words, since a small disturbance due to roundoff errors increases exponentially in unstable systems, the standard MD method cannot simulate the time-reversible behavior of the time-reversible microscopic laws.

For example, our previous studies, ${ }^{14,23}$ which are based on a "bit-reversible algorithm" for the MD method and a "controlled noise" with it, have revealed that the standard MD methods cannot avoid numerical irreversibility, since round-off errors due to floating-point real number arithmetic appear in the standard MD simulations. (Note that the bitreversible algorithm [Bit $\mathrm{MD}$ ], which was developed by Levesque and Verlet, ${ }^{24}$ is free of any round-off error and is completely time-reversible and, therefore, can detect any irreversibility in the simulations. The complete timereversibility of the Bit MD simulation has been clearly demonstrated in the works by Levesque and Verlet ${ }^{24}$ and by the present authors. ${ }^{14,23}$ ) Because of this numerical irreversibility, the standard MD method is not suitable for investigating expansion shock waves from a microscopic viewpoint. Instead, we will employ the Bit MD method for the present study since, in the Bit MD simulation, an effect of the irreversibility can be introduced in a controlled fashion. This technique will enable us to get some insight into how the expansion shock wave is formed in the present implosion process and to clarify the effect of the irreversibility on it.
In this context, in order to study the characteristics of the expansion shock wave from a microscopic viewpoint, an implosion process dual to an explosion process is investigated by means of the Bit MD method. ${ }^{25}$ The present paper is organized as follows. In Sec. II, we give a brief review of the MD techniques including the bit-reversible algorithm in order to simulate an implosion process dual to an explosion process. In Sec. III, we investigate how the expansion shock wave is formed in the implosion process and discuss the effect of the irreversibility on it. Finally, the conclusion is given.

\section{MD TECHNIQUES}

To make the present paper self-consistent, we will first give a brief review on Bit MD. ${ }^{14,23,24}$ Now, we will consider a system of $N$ particles of mass $m$ enclosed in a cubical box of size $L$, which is discretized into small cubical subcells. It is assumed that particle locations are discretized in that any particle resides on the vertexes of the cubical subcells. The minimum lattice distance $\Delta L(=L / M)$ or the size of the cubical subcell, by which the coordinate space is discretized, is defined as the side length of the cubical box $L$ divided by some integer value $M$. Although we can choose any integer value $M$, the maximum value is $2^{n}$ if $n$-bit integers are employed for the computation. Because of this discretization, the position of a particle in the discrete coordinate system is represented by integers. In this context, the equation of motion for the particles in the discrete coordinate system can be written as

$$
\begin{aligned}
\frac{\partial^{2} X_{i}}{\partial t^{2}}(\Delta t)^{2} & =X_{i}(t+\Delta t)-2 X_{i}(t)+X_{i}(t-\Delta t) \\
& =\sum_{j}\left[\left(\frac{f_{i j}(t)}{m}\right) \frac{(\Delta t)^{2}}{\Delta L}\right]_{\text {Integer }},
\end{aligned}
$$

where $f_{i j}(t)$ is a partial force exerted by the $j$ th particle on the $i$ th particle located at position $X_{i}$ at a time $t$. The term in the summation on the right-hand side of Eq. (1) is calculated based on the particle locations, and the value in the bracket [ $]_{\text {Integer }}$ is converted to an integer since, in general, the value calculated in this way is not an integer but a real number. Because of both the space discretization and this conversion process to integer, Bit MD keeps the time reversibility and is free from any round-off error. ${ }^{24}$

In the present study, we assume for simplicity the twodimensional system and integrate a set of classical equations of motion for the particles interacting through the following "repulsive" Lennard-Jones potential with a cutoff radius $r_{c}$ of $2^{1 / 6} \sigma$, in order to mimic particles in gas:

$$
\phi(r)= \begin{cases}4 \varepsilon\left[\left(\frac{\sigma}{r}\right)^{12}-\left(\frac{\sigma}{r}\right)^{6}\right], & \left(r \leqslant 2^{1 / 6} \sigma\right), \\ 0, & \left(r>2^{1 / 6} \sigma\right) .\end{cases}
$$

Here, the constants $\sigma, \varepsilon, m, \varepsilon / \kappa_{B}$, and $\sigma(m / \varepsilon)^{1 / 2}$ are chosen, respectively, as units of length, energy, mass, temperature, and time, where $\kappa_{B}$ is Boltzmann's constant. 

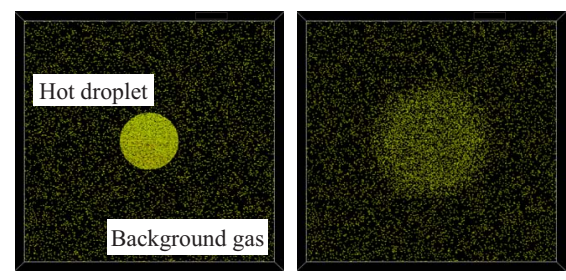

(a) $t_{E x}=0$

(b) $t_{\mathrm{Ex}}=10$

(c) $\mathrm{t}_{\mathrm{Ex}}=20$

FIG. 1. (Color online) Snapshots of the explosion simulation by the molecular-dynamics method. The locations of the particles are depicted by dots. (a) shows an initial setup for the explosion simulation. As soon as the simulation starts, the initial hot droplet starts to expand and the high-density region corresponding to a kind of compression shock wave propagates outwards.

\section{A. Initial setup for the explosion process}

In order to simulate an explosion process by means of the MD method, an initial "hot droplet" and "background gas" around it are prepared. ${ }^{25}$ In the present study, they can be prepared by cutting out a droplet from a thermally equilibrated periodic system as follows. ${ }^{26-28}$ In a square cell, $N_{\text {eq }}$ particles are equilibrated under periodic boundary conditions at a target temperature and number density by using a temperature control technique. ${ }^{26}$ From the inner region of this equilibrated system, the initial hot droplets are generated by cutting out $N_{d}$ particles included in a circular region of a radius $r_{d}$. The low-density background gas with the target temperature and number density is similarly produced, except that the particles inside a circular region of a radius $r_{d}$ are removed from the center of the square cell. Finally, the particles prepared for the hot droplet are immersed in the hole of the background gas in the square cell. The background gas together with the hot droplet immersed in the hole forms the initial setup for the simulation, ${ }^{25}$ as shown in Fig. 1. The number density $\rho$, temperature $T$, and number of particles in the hot droplet are set to be 0.80, 3.0, and 3600, respectively. As for the background gas, they are set to be $0.08, \sim 0.0$, and 7891 , respectively. Accordingly, the total number of particles $N$ becomes 11491. Technical details for the initial setup are summarized in Table I.

Note that, since the state of an initial hot droplet is one of equilibrium, the particle velocity distribution in the hot droplet becomes the Maxwell-Boltzmann distribution corresponding to the target initial temperature. Therefore, in the initial hot droplet, the velocities of each particle are determined statistically. Similarly, in the initial background gas, the velocities of each particle are determined statistically.

TABLE I. Technical details for the initial setup.

\begin{tabular}{lcc}
\hline \hline Items & Hot droplet $^{\mathrm{a}}$ & Background gas \\
\hline Number of particles & 3600 & 7891 \\
Number density $\rho\left[\sigma^{-2}\right]$ & 0.80 & 0.08 \\
Temperature $T\left[\varepsilon / \kappa_{B}\right]$ & 3.0 & $\sim 0.0$ \\
\hline \hline
\end{tabular}

${ }^{\mathrm{a}}$ The radius of the initial hot droplet $r_{d}$ is approximately $37.8[\sigma]$.

\section{B. Explosion process}

To simulate the explosion process under a restriction of "constant energy," the set of equations of motion is integrated by using Bit MD with $\Delta t=0.005$ as the time increment for the integration. The total number of time steps is set to be 4000; i.e., the terminating time for the simulation of the explosion process is set to be $t_{\mathrm{Ex}}=20$. Even though the periodic boundary conditions are imposed at the boundaries of the square cell and may affect adversely the simulation result, we try to avoid the adverse effect by using a sufficiently large square cell. To this aim, the cell length $L$ is set to be $320[\sigma]$. For this size of the cell and the present simulation time period of $t_{\mathrm{Ex}}=20$, the boundary effect does not propagate to the explosion area of our interest and, therefore, the explosion process is not affected by the boundary conditions. Note that the adverse effect caused by the discretization of the coordinate for the particles is small enough to be neglected from a physical point of view. ${ }^{14,23,24}$ In the present study, the minimum lattice distance $\Delta L$ for the 30-bit integers for the present Bit MD simulation is $3 \times 10^{-7}[\sigma]\left(=L / M=320 / 2^{30}\right)$, and is extremely small even compared with the particle diameter, $1[\sigma]$.

First of all, in order to overview a process of an explosion, snapshots of the explosion simulation result at various timings are shown in Fig. 1, where the locations of the particles are depicted by dots. As shown in Fig. 1, when the explosion simulation starts, the initial hot droplet rapidly expands, compressing the background gas. This compression generates a kind of shock wave in the background gas.

To examine the characteristics of the explosion process more clearly, radial distributions of the temperature $T$ and radial velocity $V_{r}$ are plotted in Fig. 2 . In the figure, the horizontal axis represents a radial distance from the center of the hot droplet at the initial time $t_{\mathrm{Ex}}=0$. Here, the temperature and radial velocity at the radial position $r$ are the ones averaged over circumferentially. Moreover, all the results are averaged over 10 simulations with the identically prepared initial setup. Since most particles move in the radial direction, the temperature is defined taking into account the radial velocity. (Note that, as mentioned previously, since the initial velocity distribution is set to be the Maxwell-Boltzmann distribution corresponding to the target initial temperature, the velocities of each particle are determined statistically. Therefore, at time $t_{\mathrm{Ex}}=0$, local temperature in the initial hot droplet and in the background gas fluctuates in a statistical sense.)

As shown in Fig. 2, temperature around $r \sim 47$ at $t_{\mathrm{Ex}}$ $=2$ changes discontinuously. Similarly, the radial velocity (around $r \sim 47$ ) changes discontinuously or rapidly. These discontinuities propagate outwards as time passes. Behind the discontinuous surface, the temperature and radial velocity rapidly increase. Furthermore, after the passage of the discontinuous surface, the "fluid" starts to move abruptly and is compressed. Therefore, it is expected that this discontinuous surface corresponds to the so-called compression shock wave from a fluid dynamical point of view. After the rapid change behind the discontinuous surface, i.e., around $r=20-38$, temperature decreases gradually because of an adiabatic-like expansion. 

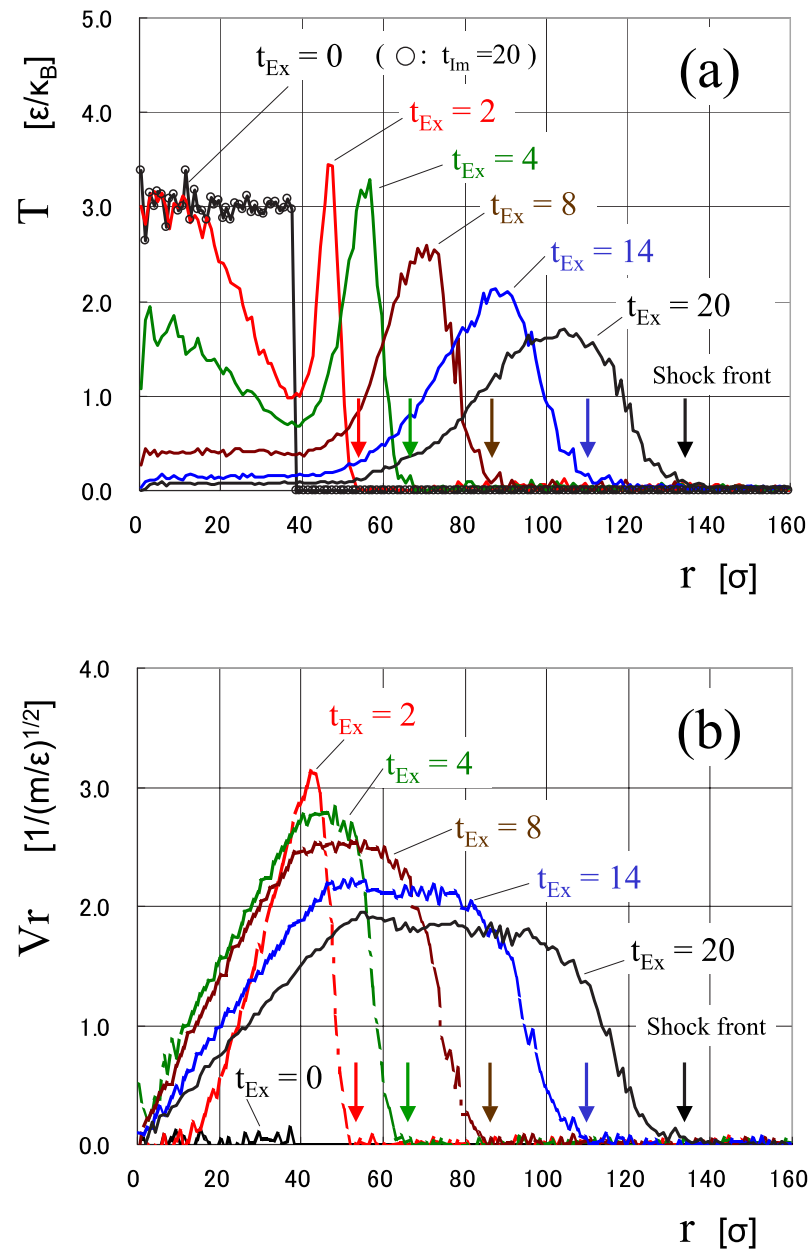

FIG. 2. (Color online) Radial distributions of the temperature and radial velocity in the explosion process. The top and bottom figures represent (a) temperature $T$ and (b) radial velocity $V_{r}$, respectively. The arrow in each profile represents a shock location. The temperature and radial velocity at the radial position $r$ are the ones averaged over circumferentially. All the results are averaged over 10 simulations with the identically prepared initial setup. Note that, in (a), open circles $(O)$ represent the simulation result of the implosion process at $t_{\mathrm{Im}}=20$ in the case of $N_{\mathrm{cn}} / N=0 \%$ (i.e., without noise). See Sec. III for details of the implosion process.

We have confirmed that the explosion simulation generates a discontinuous surface corresponding to a kind of compression shock wave, as has been described in previous works. ${ }^{19-22,29,30}$

\section{Implosion process dual to explosion process}

In order to simulate the implosion process in the Bit MD method, we make use of the explosion simulation result at the final time (i.e., $t_{\mathrm{Ex}}=20$ ) and conduct a time-reversal operation on it. At the time-reversal operation, the initial positions and velocities of the particles are set to be the ones at $t_{\mathrm{Ex}}=20$ in the explosion simulation, except that all the particles reverse their velocities. Taking this setup as the initial condition, the implosion simulation starts. Then, it is expected that a kind of compression shock wave turns into an expansion shock wave in this simulation, since the Bit MD simulation is completely time-reversible. Note that, in the present study, a discontinuous wave appearing in moleculardynamics simulations is also called a shock wave.
In this paper, in order to investigate the characteristics of the expansion shock wave, the "controlled noise" is added to the implosion simulation mentioned above. This is because it is expected that the expansion shock wave is unstable and is affected by the irreversibility of the system, which is induced by the controlled noise. The present controlled noise is a deliberate displacement of the particles; that is, the particles suffer displacement of their position suddenly by a certain amount, in a random direction. Here, the "quantity" of the noise is controlled in the following three ways: ${ }^{14,23}$

(1) The magnitude of the displacement $d X_{\mathrm{cn}}$.

(2) The frequency of addition, $F_{\mathrm{cn}}$, of the displacement.

(3) The number of particles, $N_{\mathrm{cn}}$, added with the displacement.

To parametrically control the number of particles affected by the noise, $N_{\mathrm{cn}} / N$ is set to be $0 \%, 1 \%, 10 \%$, or $100 \%$, where $N$ represents the total number of particles. On the other hand, $d X_{\mathrm{cn}}$ and $F_{\mathrm{cn}}$ are fixed; i.e., the magnitude of the displacement $d X_{\text {cn }}$ is set to be $1[\Delta L]$, and the frequency of its addition $F_{\text {cn }}$ is for every time step. The magnitude of this displacement is the minimum among the possible ones and is extremely small $\left(3 \times 10^{-7}[\sigma]\right)$ compared with even the particle diameter, $1[\sigma]$.

\section{RESULTS}

In this section, we will discuss the implosion phenomenon and investigate a kind of expansion shock wave possibly generated in the dual implosion simulation. The radial distributions of the temperature and radial velocity are shown in Fig. 3. In these figures, the distributions of the temperature and radial velocity at $t_{\mathrm{Im}}=6,12,16,18$, and 20 correspond to the ones at $t_{\mathrm{Ex}}=14,8,4,2$, and 0 in the explosion simulation, respectively. The horizontal axis represents the radial distance from the center of the hot droplet at the initial time $t_{\mathrm{Ex}}=0$ in the explosion simulation. In those figures, we will first focus on the simulation result with $N_{\mathrm{cn}} / N=0 \%$, since it corresponds to the time-reversible Bit MD simulation result. As we expect, each distribution agrees exactly with the corresponding distribution of the explosion process shown in Fig. 2, which is demonstrated by replotting the temperature distribution at $t_{\mathrm{Im}}=20$ in Fig. 2(a). In the figure, the temperature distribution at $t_{\mathrm{Im}}=20$, which is designated by open circles, agrees exactly with the initial temperature distribution for the explosion simulation. The only exception is that the velocity reverses its direction, as shown in Figs. 2(b) and 3(b). That is, since the process is an implosion, the medium implodes inwards. The discontinuous wave observed in the explosion process propagates inwards, instead, keeping its characteristics of discontinuity. Therefore, this wave is a kind of shock wave. After passage of the discontinuous wave, the propagation medium is suddenly decelerated to zero velocity and the temperature suddenly decreases. In other words, this shock wave is an expansion wave. Since the propagation medium expands after the passage of the shock wave, we can conclude that a kind of expansion shock wave is formed in the implosion simulation by Bit MD. 


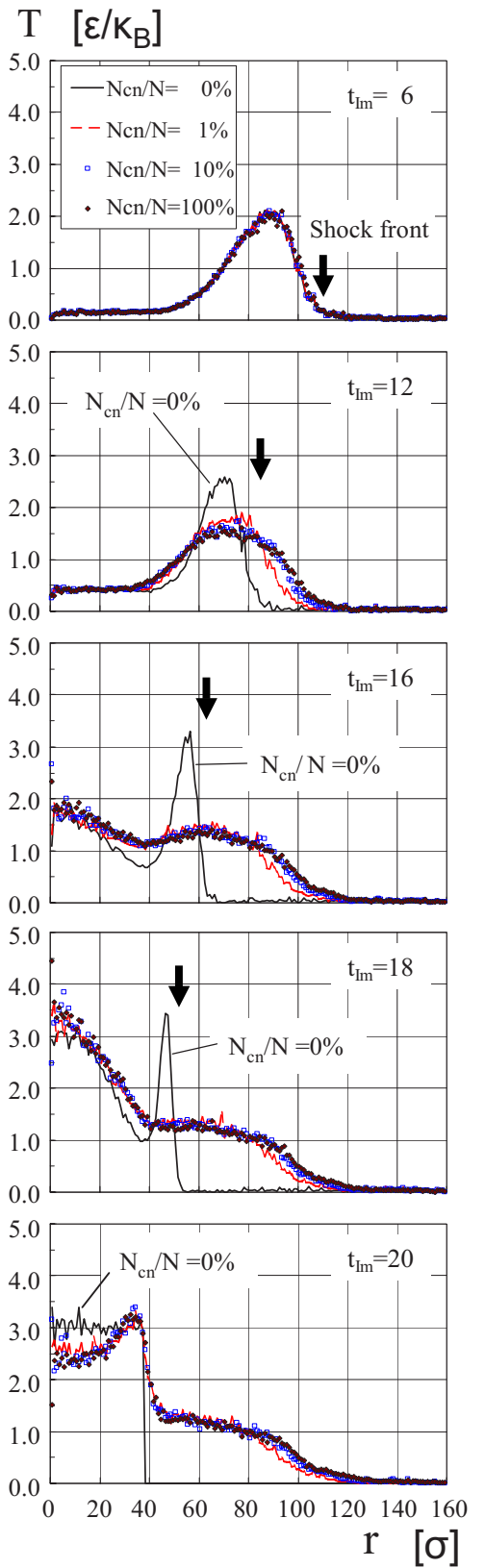

(a) Temperature: $\mathrm{T}$
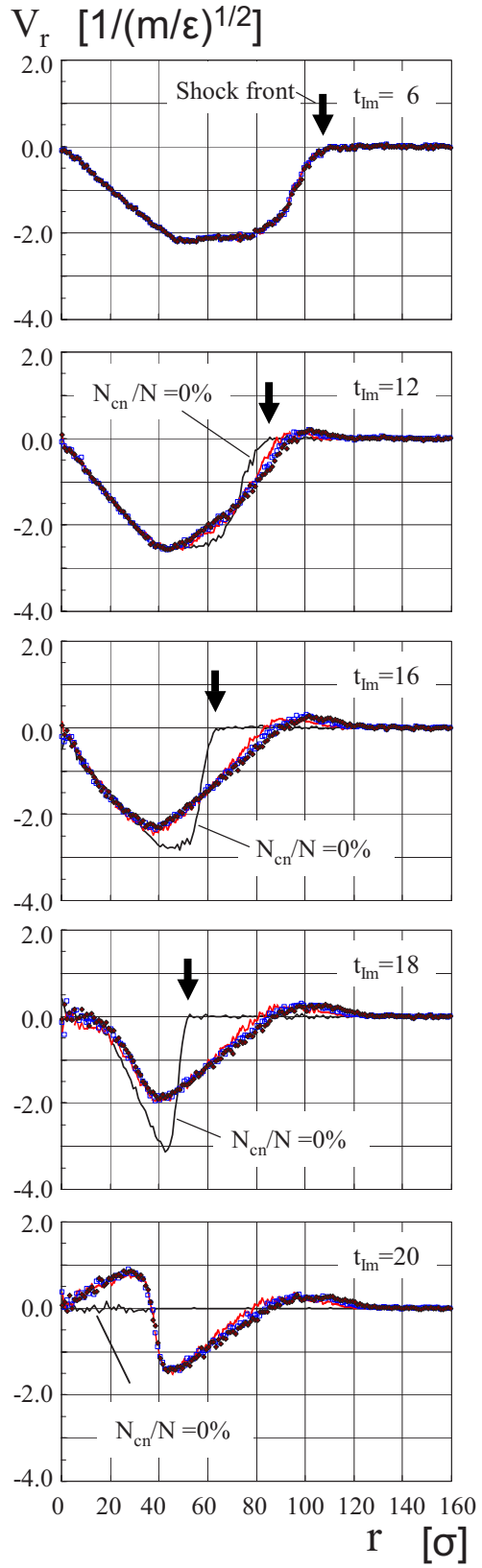

(b) Radial velocity: $\mathrm{V}_{\mathrm{r}}$
FIG. 3. (Color online) Radial distributions of the temperature and radial velocity in the implosion process. The left and right figures represent (a) temperature and (b) radial velocity, respectively. $N_{\mathrm{cn}} / N$ represents a quantity of the controlled noise added to the simulation. Here, $N_{\text {cn }} / N=0 \%$ (i.e., without noise) is the timereversible Bit MD simulation result. The arrow in each figure represents a shock location of an expansion shock wave in the case of $N_{\mathrm{cn}} / N=0 \%$. As time goes on (i.e., from the top figures to the bottom figures), the medium propagates inwards. In the case of $N_{\mathrm{cn}} / N$ $=0 \%$, a discontinuous surface corresponding to an expansion shock wave is formed. However, a kind of expansion shock wave suddenly disappears due to the controlled noise, even in the case of $N_{\mathrm{cn}} / N=1 \%$. All the results are averaged over 10 simulations with the identically prepared initial setup.
In our earlier papers, ${ }^{14,23}$ we demonstrated that the controlled noise can give rise to an irreversible nature in the Bit MD simulation and we examined in detail the effect of the controlled noise on the irreversibility. Therefore, by adding some controlled noise in the present simulation, we can examine an effect of the irreversibility on the expansion shock wave quantitatively. The effects of the controlled noise are depicted in Fig. 3. As shown in these figures, the discontinuous surface or the expansion shock wave dramatically disappears due to the controlled noise, even in the case of $N_{\text {cn }} / N=1 \%$. For example, at $t_{\mathrm{Im}}=12-18$ in Fig. 3(a), the peak of the discontinuous surface of the temperature is degraded dramatically due to even the small noise in the case of $N_{\mathrm{cn}} / N=1 \%$. Even though further controlled noise is added to the implosion process, the behavior of the process is not influenced so much. That is, the behavior of the process con- verges into a single one regardless of the "quantity" of the noise. In other words, the behavior of the present implosion process is dramatically dominated by whether the controlled noise exists.

This dramatic modification of the flow in the case of the dual implosion process shows a sharp contrast to that in the case of the explosion process. That is, in the case of the explosion process, no such modification is induced by the controlled noise, which is consistent with the widely accepted view.

Let us examine the behavior of the implosion process modified by the irreversibility from a macroscopic viewpoint. The region of expansion in the flow can be clearly identified as a region in the velocity distribution where the velocity recovers to zero from a negative peak value. In the behavior of the implosion process simulated by Bit MD with 
the controlled noise, the region of expansion becomes more diffusive compared to the one simulated by Bit MD without the controlled noise. Furthermore, the diffusive expansion region in the former becomes more diffusive as time goes on (until $t_{\mathrm{Im}}=16$ ), while the one in the latter keeps its form and remains discontinuous since it is a kind of expansion shock wave. Therefore, we can interpret the diffusive expansion region as a so-called isentropic expansion wave. This behavior of the implosion process is natural from the viewpoint of macroscopic fluid dynamics.

\section{CONCLUSION}

In order to study the characteristics of the expansion shock wave from a microscopic viewpoint, an implosion process dual to an explosion process was investigated by means of the Bit MD method. It was demonstrated that a kind of expansion shock wave, which was formed in the timereversible simulation, was sensitive to the noise added to the simulation. Since the noise added to the Bit MD simulation causes the irreversibility in the simulation, we can conclude that the irreversibility causes the disappearance of the expansion shock wave. Furthermore, the macroscopic flow behavior caused by the noise was independent of the "quantity" of the noise. In other words, the behavior of the macroscopic implosion process is dramatically dominated by whether the controlled noise exists, i.e., the irreversibility. This may correspond to the fact that the expansion shock wave does not exist in a gaseous medium in nature, and may verify a widely held belief that, because of the second law of thermodynamics, we cannot observe the expansion shock wave in a gaseous medium in nature.

${ }^{1}$ Ya. B. Zel'dovich and Yu. P. Raiser, Physics of Shock Waves and HighTemperature Hydrodynamic Phenomena (Dover, New York, 2002).

${ }^{2}$ J. Zel'dovich, "Propagation of shock waves in a gas in the presence of a reversible chemical reaction," Zh. Eksp. Teor. Fiz. 16, 365 (1946).

${ }^{3}$ P. A. Thompson, "A fundamental derivative in gas dynamics," Phys. Fluids 14, 1843 (1971).

${ }^{4}$ P. A. Thompson and K. S. Lambrakis, "Negative shock waves," J. Fluid Mech. 60, 187 (1973)

${ }^{5}$ A. A. Borisov, Al. A. Borisov, S. S. Kutateladze, and V. E. Nakoryakov, "Rarefaction shock wave near the critical liquid-vapour point," J. Fluid Mech. 126, 59 (1983).

${ }^{6}$ A. G. Ivanov and S. A. Novikov, "Rarefaction shock waves in iron and steel," Sov. Phys. JETP 13, 1321 (1961).
${ }^{7}$ R. Menikoff and B. J. Plohr, "The Riemann problem for fluid flow of real materials," Rev. Mod. Phys. 61, 75 (1989).

${ }^{8}$ J. W. Bates and D. C. Montgomery, "Some numerical studies of exotic shock wave behavior," Phys. Fluids 11, 462 (1999).

${ }^{9}$ N. M. Bulgakova, "Possibility of rarefaction shock wave under short pulse laser ablation of solids," Phys. Rev. E 60, R3498 (1999).

${ }^{10}$ N. M. Bulgakova, I. M. Bourakov, and N. A. Bulgakova, "Rarefaction shock wave: Formation under short pulse laser ablation of solids," Phys. Rev. E 63, 046311 (2001).

${ }^{11}$ J. L. Lebowitz, "Boltzmann's entropy and time's arrow," Phys. Today 46(9), 32 (1993).

${ }^{12}$ W. G. Hoover, Time Reversibility, Computer Simulation, and Chaos (World Scientific, Singapore, 1999).

${ }^{13}$ J. E. Broadwell, "Irreversibility in a reversible lattice gas," J. Stat. Phys. 103, 1125 (2001).

${ }^{14}$ N. Komatsu and T. Abe, "Numerical irreversibility in time-reversible molecular dynamics simulation," Physica D 195, 391 (2004).

${ }^{15}$ J. H. Lee and B. H. K. Lee, "Cylindrical imploding shock waves," Phys. Fluids 8, 2148 (1965).

${ }^{16}$ Y. Nakamura, "Analysis of self-similar problems of imploding shock waves by the method of characteristics," Phys. Fluids 26, 1234 (1983).

${ }^{17}$ M. Yousaf, "Imploding spherical and cylindrical shocks," Phys. Fluids 29, 680 (1986).

${ }^{18} \mathrm{H}$. Matsuo and K. Fujiwara, "Explosive-driven cylindrical imploding shocks," Phys. Fluids A 2, 266 (1990).

${ }^{19}$ P. Gaspard and J. Lutsko, "Imploding shock wave in a fluid of hard-core particles," Phys. Rev. E 70, 026306 (2004).

${ }^{20}$ W. G. Hoover, "Structure of a shock-wave front in a liquid," Phys. Rev. Lett. 42, 1531 (1979).

${ }^{21}$ B. L. Holian, W. G. Hoover, B. Moran, and G. K. Straub, "Shock-wave structure via nonequilibrium molecular dynamics and Navier-Stokes continuum mechanics," Phys. Rev. A 22, 2798 (1980).

${ }^{22} \mathrm{E}$. Salomons and M. Mareschal, "Usefulness of the Burnett description of strong shock waves," Phys. Rev. Lett. 69, 269 (1992).

${ }^{23} \mathrm{~N}$. Komatsu and T. Abe, "Noise-driven numerical irreversibility in molecular dynamics technique," Comput. Phys. Commun. 171, 187 (2005).

${ }^{24}$ D. Levesque and L. Verlet, "Molecular dynamics and time reversibility," J. Stat. Phys. 72, 519 (1993).

${ }^{25} \mathrm{~N}$. Komatsu and T. Abe, "Why does not expansion shock wave exist in nature?" in Proceedings of the 25th International Symposium on Rarefied Gas Dynamics, Russia, July 2006.

${ }^{26}$ N. Komatsu and T. Abe, "Shape effect of hot droplets on fragmentation," Phys. Rev. E 72, 021601 (2005).

${ }^{27}$ A. Vicentini, G. Jacucci, and V. R. Pandharipande, "Fragmentation of hot classical drops," Phys. Rev. C 31, 1783 (1985).

${ }^{28}$ A. Strachan and C. O. Dorso, "Time scale in fragmentation," Phys. Rev. C 55, 775 (1997).

${ }^{29}$ T. E. Itina, J. Hermann, P. Delaporte, and M. Sentis, "Laser-generated plasma plume expansion: Combined continuous-microscopic modeling," Phys. Rev. E 66, 066406 (2002).

${ }^{30}$ F. Garrelie, J. Aubreton, and A. Catherinot, "Monte Carlo simulation of the laser-induced plasma plume expansion under vacuum: Comparison with experiments," J. Appl. Phys. 83, 5075 (1998). 